\title{
Virus-mediated hijack of one-carbon metabolism
}

Epstein-Barr virus (EBV) infection mimics physiological signals to drive B cell growth and survival. In a recently published article, Wang et al. investigated the early metabolic changes associated with EBV infection. They found an early upregulation of one-carbon (1C) metabolism mediated by the viral oncoprotein EBNA2 that is critical for EBV-induced rapid B cell growth and that is similarly observed upon physiological stimulation of $B$ cell expansion.

EBV is a gammaherpesvirus whose infection is linked to many cancers, including several $B$ cell malignancies in which it can drive uncontrolled B cell growth. Indeed, a hallmark of EBV is its ability to transform primary human B cells into hyperproliferative blasts. This transformation necessitates an extensive remodelling of the host metabolic pathways. However, the systemic effects of EBV infection on B cell metabolism and the mechanisms through which the viral proteins modulate the host metabolism are poorly known. Therefore, Wang et al. set out to characterize EBV-driven metabolic reprogramming of B cells.

Wang et al. used primary human $\mathrm{CD} 19^{+} \mathrm{B}$ cells isolated from human donors and infected them in vitro with a patient-isolated strain of EBV. They quantitatively measured the temporal proteomic changes between infection and generation of fully transformed lymphoblastoid cell lines (LCLs). They found several clusters of $B$ cell proteins with distinct temporal expression patterns. At 4 days post infection (DPI) the clusters of upregulated proteins were shown to be enriched for proteins belonging to metabolic pathways, including 1C metabolism. Therefore, the authors hypothesized that EBV infection could be associated with an early metabolic rewiring.
Examining this in more detail, the authors found an upregulation of all glycolytic enzymes at 2 DPI. Consistent with an enhanced glycolytic flux, $B$ cell glucose consumption and lactate release were increased by 2 DPI. Culturing of cells in media containing galactose instead of glucose strongly impaired EBV-mediated B cell outgrowth, suggesting that glucose is an essential carbon source for EBV-driven transformation.

The authors also reported early changes in mitochondrial proteins. In particular, they observed an upregulation of many electron transport chain (ETC) components, which was associated with enhanced oxygen consumption rate. ETC inhibition impaired EBV-driven B cell outgrowth, suggesting that enhanced oxidative phosphorylation was necessary to support EBV-driven transformation. In addition, the authors observed a robust upregulation of mitochondrial $1 \mathrm{C}$ enzymes at 2 DPI, including MTHFD2, a 1C metabolic enzyme rarely expressed by adult cells. Such an upregulation of mitochondrial 1C enzymes was also observed upon stimulation of B cells with prototypical agonists functioning in lymph node germinal centre reactions, suggesting it may have a relevant role in B cell growth.

Looking into this pathway further, the authors reported an increase in serine consumption over the first week of infection associated with EBNA2-mediated upregulation of the major serine transporters. Extracellular serine was catabolized through the mitochondrial 1C pathway and was required to sustain EBVdriven primary B cell outgrowth. Providing more support for the essential role of $1 \mathrm{C}$ metabolism upregulation, pharmacological inhibition of the pathway, either

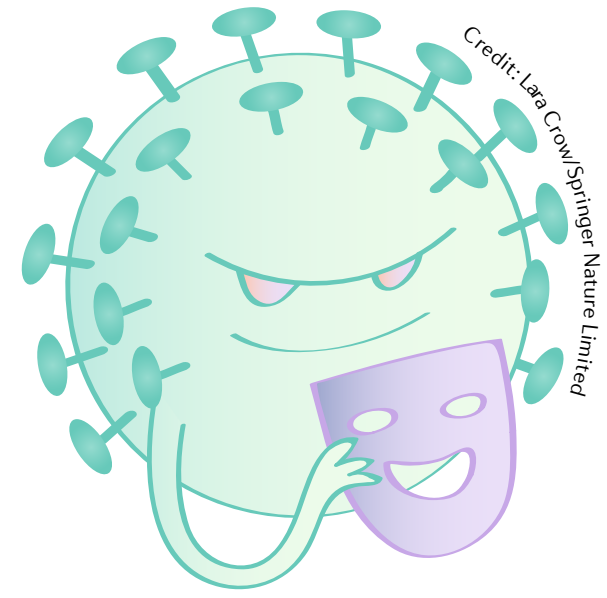

in newly infected B cells or fully transformed LCLs, significantly diminished proliferation. Knockout of MTHFD2 alone was sufficient to diminish LCL proliferation, suggesting a critical role for this enzyme. Interestingly, the authors showed that MTHFD2 expression was driven transcriptionally by EBV-encoded protein EBNA2 and MYC instead of being driven by its physiological activator ATF4.

Functionally, induction of mitochondrial 1C metabolism was shown to be an important source of intramitochondrial $\mathrm{NADPH}$, which is required to sustain proliferation presumably by providing sufficient reducing power to support the increased activity of anabolic pathways. Additionally, 1C metabolism was shown to provide $1 \mathrm{C}$ units to support nucleotide synthesis to sustain the energetic ATP demands of the infected cells and be heavily utilized for glutathione biosynthesis providing cell redox defences.

These findings highlight the potential for inhibition of $1 \mathrm{C}$ metabolism as a therapeutic approach in EBV-driven lymphomas. In particular, MTHFD2 might be an ideal therapeutic target with limited toxicity, given that this enzyme is not expressed by most adult cells.

Maria Giuseppina Baratta, Senior Editor, Nature Communications

ORIGINAL ARTICLE Wang, L. W. et al. EpsteinBarr-virus-induced one-carbon metabolism drives B cell transformation. Cell Metab.

https://doi.org/10.1016/j.cmet.2019.06.003 (2019) 